\title{
Mechanism and Research Methods on the Integration of Cultural Industry and Tourism Industry
}

\author{
Jinghui Qu \\ School of Management, Liaoning Institute of Science and Technology, Benxi, Liaoning, 117004, \\ China \\ email: bctesorg@163.com
}

\begin{abstract}
Keywords: Culture Industry, Tourism industry, Industry convergence, Research methods Abstract. Under the background of economic globalization, the rapid development of high technology to promote industrial integration. The unique creativity of cultural industry and the cultural communication of tourism industry provide a good foundation for the development of the integration of the cultural industry. This paper selects 31 China provincial administrative divisions (except Hong Kong, Macao and Taiwan). According to the geographical position will be divided into the northeast, North China, East China, central China, Southern China, northwest, southwest of the 7 regions were analyzed (Table 1). and establish the index system to determine the research area and data sources, and put forward the research methods of cultural industry tourism industry. This provides a theoretical support for the analysis of spatial and temporal changes in the integration of cultural industry and tourism industry, which is of great significance to the development of tourism $+\mathrm{X}$ industry.
\end{abstract}

\section{Introduction}

Domestic and foreign scholars conduct the research on the cultural industry and tourism industry integration from different perspectives. Foreign scholars research mainly from the perspective of heritage protection and human intercultural communication, related to the form of cultural tourism products[1,2], tourism development of cultural resources[3] and the effect of development of cultural and tourism[4] and other aspects. Domestic scholar Cheng Xiaoli and others put forward by means of qualitative analysis a series of promoting measures on the culture industry and tourism industry integration in Anhui province, in the view of government, industry and market[5]; Xin xin classified and summarized ways of cultural and tourism industry convergence, and put forward the integrated development model of community tourism in Kaifeng according to local conditions [6]; Wu qian analyzed qualitatively the integration mechanism of the cultural industry and tourism industry in Guizhou province with an idea of characteristic development model based on the creativity of cultural industry and the cultural transmission of tourism industry[7]; Because current researches are conducted more based on qualitative analysis and in a single research area, quantitative comparison research in multiarea is less. This paper, based on a sample of 7 major areas in China's 31 provincial administrative divisions(except Hong Kong, Macao and Taiwan), constructs the index system of the evaluation of the integration level of the cultural industry and the tourism industry and the convergence mechanism and research methods by using the principle of the coordination theory.

\section{The Mechanism of the Integration of Cultural Industry and Tourism Industry}

In the developing process, culture industry and tourism industry are mutually dependent, integrated and promoted. In recent years, cultural and tourism consumer market demand is becoming more complex, comprehensive and individual with the improvement of industrial efficiency together with science and technology. Culture, tourism and related industry enterprises has formed a cooperation or competition relationship, which provides driving force for the integration of cultural industry and tourism industry. Culture enriches the connotation of tourism resources, providing material for the development of tourism projects, attracting more tourists to participate in tourism activities; and the development of tourism activities enhances the spread of culture. Cultural products 
and services link various value chains of the tourism industry through consumers. Tourists from different regions become the carrier of cultural transmission, spreading the local cultural resources effectively ( Fig.1).

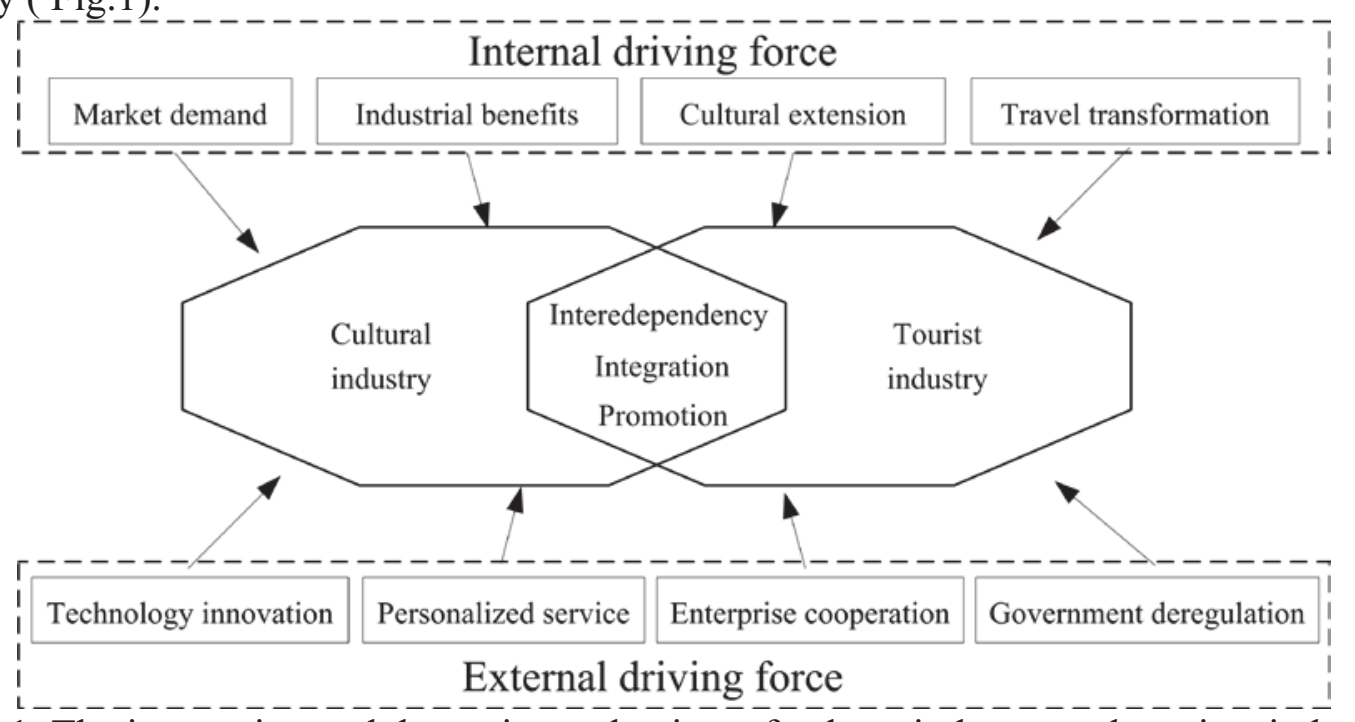

Fig. 1. The integration and dynamic mechanism of culture industry and tourism industry

\section{Research Methods of the Integration of Cultural Industry and Tourism Industry}

Study Area Selection. This paper has selected 31 provincial administrative divisions of China(except Hong Kong, Macao and Taiwan) for evaluation and analysis according to their geographical position, including the northeast(Liaoning, Jilin, Heilongjiang), north China(Beijing, Tianjin, Hebei, Shanxi, Inner Mongolia), east China(Shandong, Jiangsu, Anhui, Zhejiang, Fujian, Shanghai, Jiangxi), central China(Hubei, Hunan, Henan ), south China(Guangdong, Guangxi, Hainan), northwest(Ningxia, Xinjiang, Qinghai, Shaanxi, Gansu ), southwest(Sichuan, Yunnan, Guizhou, Tibet, Chongqing ).

Establishment of Index System.Quantitative studies on the integration of cultural industry and tourism industry by Chinese scholars are less. And selection of evaluation indicators of the level of industrial convergence is not perfect. This research is based on the previous research results to settle the problems in the development of industrial convergence. The cultural industry and tourism industry alignment assessment system is built on the basis of products, markets, technology and talents according to the availability of data. On the whole, the system is made of cultural industry development and the development of tourism industry. Industrial development system follows the principle of from the overall strength to the local development, employs 6 first level indicators and 20 second level indicators including cultural (Tourism) industry comprehensive strength, Cultural (Tourism) industry operating income and cultural (tourism) industry organizations in general (Table 2).

Data Sources. Based on data availability and manipulability, the cultural industry data in this study are from "China Statistical Yearbook of Cultural Heritage"(2004 2014), the tourism industry data from"China Tourism Statistics Yearbook"(2004 2014), statistical Yearbook and tourism statistical bulletin of provinces and cities.

Research Methods. Index Weights. In this study, index weight is determined through subjective and objective integrated weighting method. Subjective weighting method is the Delphi method---back to back scoring method by experts; entropy method is objective weighting method, which calculates the entropy of each index according to the degree of variation of each index, using information entropy, then the right of each index is corrected by entropy to arrive at a more objective index weight, which avoids the overlap between multiple indicator variables and artificial errors in determining the weight. Comprehensive weight determination is the right to determine the objective and subjective respectively by multiplication synthesis normalization to strike.

The main steps are as follows:

Raw data normalized (Equation 1) 


$$
\begin{gathered}
r_{i j}=\frac{x_{i j}-\operatorname{MIN}_{j}\left\{x_{i j}\right\}}{\operatorname{MAX}\left\{x_{i j}\right\}-\operatorname{MIN}\left\{x_{i j}\right\}} \text { (Index greatly gifted) } \\
r_{i j}=\frac{\operatorname{MAX}_{j}\left\{x_{i j}\right\}-x_{i j}}{\operatorname{MAX}_{j}\left\{x_{i j}\right\}-\operatorname{MIN}\left\{x_{i j}\right\}} \text { (Small index is excellent) }
\end{gathered}
$$

Normalized post matrix $\mathrm{R}=(r)_{m^{*} n}, r_{i j}$ is the $\mathrm{j}$-th standard evaluation object value in the $\mathrm{i}$-th evaluation index, $r_{i j} \in[0,1]$.

Calculate the entropy (Equation 2-4)

$$
\begin{gathered}
f_{i j}=r_{i j} / \sum_{j=1}^{n} r_{i j} \\
k=1 / \ln n \\
H_{i}=-k \sum_{j=1}^{n} f_{i j} \ln f_{i j}
\end{gathered}
$$

When $f_{i j}=0$, let $f_{i j} \ln f_{i j}=0$

determine the entropy (Equation 5)

$$
w_{i}=\frac{1-H_{i}}{m-\sum_{i=1}^{m} H_{i}}
$$

When $0 \leq w_{i} \leq 1, \quad \sum_{i=1}^{m} w_{i}=1$.

Industrial Integration Measure. Industrial integration refers to the interactive coupling process of the internal elements or subsystems of different industries. Industrial coordination degree reflects the degree of coupling between the interaction of different industries[8]. In this study, the coordination degree model is adopted to calculate the degree of integration of cultural industry and tourism industry, drawing on the capacity of the coupling coefficient physics model to calculate the level of integration.

(1)The total evaluation function (Equation 6):

$$
f_{\text {E或 }}=\sum_{\mathrm{i}=1}^{\mathrm{m}} w_{i} r_{i}
$$

Calculating $f_{E}$ and $f_{C}$. Both are the level of development of comprehensive evaluation of the development systems of cultural industries and tourism industry at time t.

(2)The degree of coupling (Equation 7):

$$
E C=2\left\{\left(f_{(E, t)} * f_{(C, t)}\right) /\left[\left(f_{(E, t)}+f_{(C, t)}\right)^{2}\right]\right\}^{\frac{1}{2}}
$$

Calculating the degree of coupling EC of cultural industry and tourism industry development systems at time t, .

(3)The degree of coordination:

Since the degree of coupling only reflects the degree of coupling between the cultural industry and tourism industry, not the actual level of development of cultural industry and tourism industry, the results are lack of comparability. In order to overcome this shortcoming, formula (8) is needed to calculate the regional comprehensive development level $\mathrm{F}$ (given cultural industry and tourism industry have equal influence on the level of the comprehensive development of the region):

$$
\begin{gathered}
F=\left(f_{(E, t)}+f_{(C, t)}\right) / 2 \\
D_{t}=\left(E C^{*} F\right)^{\frac{1}{2}}, \quad D_{t} \in(0,1)
\end{gathered}
$$


Calculating industrial coordination degree $D_{t}$ (Equation 9). When $D_{t}$ tends to 1 , effective coordination of development is reached between cultural industry and tourism industry or inside the elements, the system will tend to a new ordered structure; conversely, when $D_{t}$ tends to 0 , industrial coordination degree is smaller. It is independent of the state between the two systems or inside of the elements, and the system will develop disorderly.

\section{Conclusion}

(1)Improve the Integration Index of Cultural Industry and Tourism Industry, Promote Regional Economic Development.

(2)Analyze Continuously the Spatial Variation of Factors Affecting the Industrial Convergence, Find the Dominant Factor in Different Periods, and Develop Appropriate Industrial Policies.

(3)Increase the Government's Guidance and Support, Create Cultural Tourism Products with Regional Characteristics.

\section{Acknowledgement}

In this paper, the research was sponsored by the Project source:Liaoning Institute of Science and Technology service local economic innovation and development project: Strategy Research of Innovative Development of Tourism Industry in Benxi (Project No. 2016rkx-03) in 2016.

\section{References}

[1]Grey Richards, Crisp Raymond. Creative tourism. ATLAS News, 1996(23):16-20.

[2]Calvin Taylor. Beyond advocacy. Developing an evidence base for regional creative Industry strategies. Cultural Trends, 1999(3).

[3]Chhabra Aitchison, Ton Evans. The cultural relics and a model of sustainable regeneration: manufacturing "pop" in the Rhondda valleys of south wales. Managing Leisure, 2003(7):128 - 130.

[4]Antonia Besculides, Martha E Lee, Peter JMc Cormick. Residents' perceptions of the cultural benefits of tourism. Annals of Tourism Research, 2002,29(2):303-319.

[5]Cheng Xiaoli, Zhu Yawen. The integration of tourism industry and culture industry in Anhui province. Economic Geography, 2012, 32(9): 161-165.

[6]Xin Xin. Study on the convergence of cultural industry and tourism industry: mechanism, path and mode-Taking Kaifeng as an example. Kaifeng: Henan university, 2013: 39.

[7] Wu Qian. Research on cultural industry and tourism industry integration development in ethnic areas-taking Guizhou province as an example. Guizhou Ethnic Studies, 2012, 33(6): 124-127.

[8]Haken H. Erfolgsgeheimnisse Der Natur Synergetik: Die Lehre Vom Zusammenwirken. Trans. by Ling Fuhua. Shanghai: Shanghai Century Publishing Group, 2005. 